\title{
Combination Drug Therapy for Benign Prostatic Hyperplasia (BPH)
}

Author: Oliech J.S. FRCS, Affiliation: Department of Surgery, University of Nairobi. P.O. Box 30197-00100 Email Correspondence: jsoliech@yahoo.com.

\section{Summary}

\section{Background}

The incidence of benign prostatic hyperplasia peaks $80 \%$ in old age. Bothersome symptoms and progression to acute urinary retention and need for surgery are important concerns. Apha blockers and 5 alpha reductase inhibitors address these to variable extents. This article reviews the current place of medical therapy in the treatment of $\mathrm{BPH}$, with a focus on combination therapy.

\section{Data Source}

A medline literature search was performed to identify original studies including global multi-center trials and reviews on the subject.

\section{Conclusion}

$\mathrm{BPH}$ symptom reduction and shrinkage of prostate size by the use of a combination of alpha adrenergic uroselective blocker (Tamsulosin) and 5 alpha iso-enzyme inhibitor (Dutasteride) is a first option of therapy in the management of BPH especially in those cases that are surgical risks with the category of mild to moderate prostate symptom scores.

\section{Introduction}

Benign prostatic hyperplasia $(\mathrm{BPH})$ is the most common cause of urinary bladder outflow obstruction (BOO) in men aged 50 years and above(1). Patient age, androgenic stimulus, genetic and some environmental factors are etiological determinants. The incidence of the disease is about $14 \%$ in men 40 years to 49 years of age but prevalence rises to about $80 \%$ in men older than 70 years of age (2-4). The active form of testosterone, the dihydrotestosterone (DHT) stimulates prostatic cellular proliferation with attendant reduction in programmed cell death (apoptosis) (5-6). This results in increased prostatic size. The cellular proliferation, concentrated in the peri - urethral and transitional zones, is accompanied by increase in the stromal content (smooth muscles) and this combination results in symptoms of bladder outlet obstruction (Fig 1) manifesting with voiding/ obstructive and storage/irritative symptoms in various combinations $(1,6)$ Other associated symptoms are abdominal pain, haematuria, haemospermia, and erectile dysfunction.

The progression of BPH and worsening of symptoms results in acute retention of urine (AUR) and demand for BPH related surgery (7). The risk of AUR is $23 \%$ in a 60 year old by the time he becomes 80 years old $(2,3)$. This also increases morbidity and mortality of $\mathrm{BPH}$. The need to halt the progression has given impetus to the development of effective medical therapies. But to know for which group of patients the therapies would be most efficacious, it is necessary to stratify them. Two factors reported to predict the progression to AUR include prostatic volume $>30 \mathrm{cc}$ and serum prostatic specific antigen $>1.5 \mathrm{ng} / \mathrm{ml}$ and $<10.0 \mathrm{ng} / \mathrm{m}$. Patients outside this range may not benefit from the medical therapies (8).

\section{Drug Treatment Of BPH}

The discussion on medical therapy focuses on the merits of monotherapy versus combination drug therapy. Two main lines of medical therapies involve the use of alpha-adrenergic blockers and $5-\alpha$ reductase inhibitors (5). Medical therapy is a first option of treating patients with mild to moderate lower urinary symptoms (LUTs). Apart from symptom resolution, these therapies can further stop the disease progression to acute retention of urine (AUR) or BPH-related surgeries with the attendant complications (9).

Alfuzocin and tamsulosin are uro-selective (acts only on $\alpha 1$ a and $\alpha 1 \mathrm{~d}$ adrenergic subtypes found in prostatic capsule, prostatic smooth muscle, bladder neck smooth muscle (10-13). The drugs act by reducing muscle tone. At the level of the bladder neck, reduction of muscle tone will improve the outlet obstruction in patients with BPH (Fig. 2). Towards this end, the proportion of the stromal tissue to the epithelial glandular tissue is a key 


\section{Combination Drug Therapy for Benign Prostatic Hyperplasia (BPH)}

\section{Oliech J.S.}

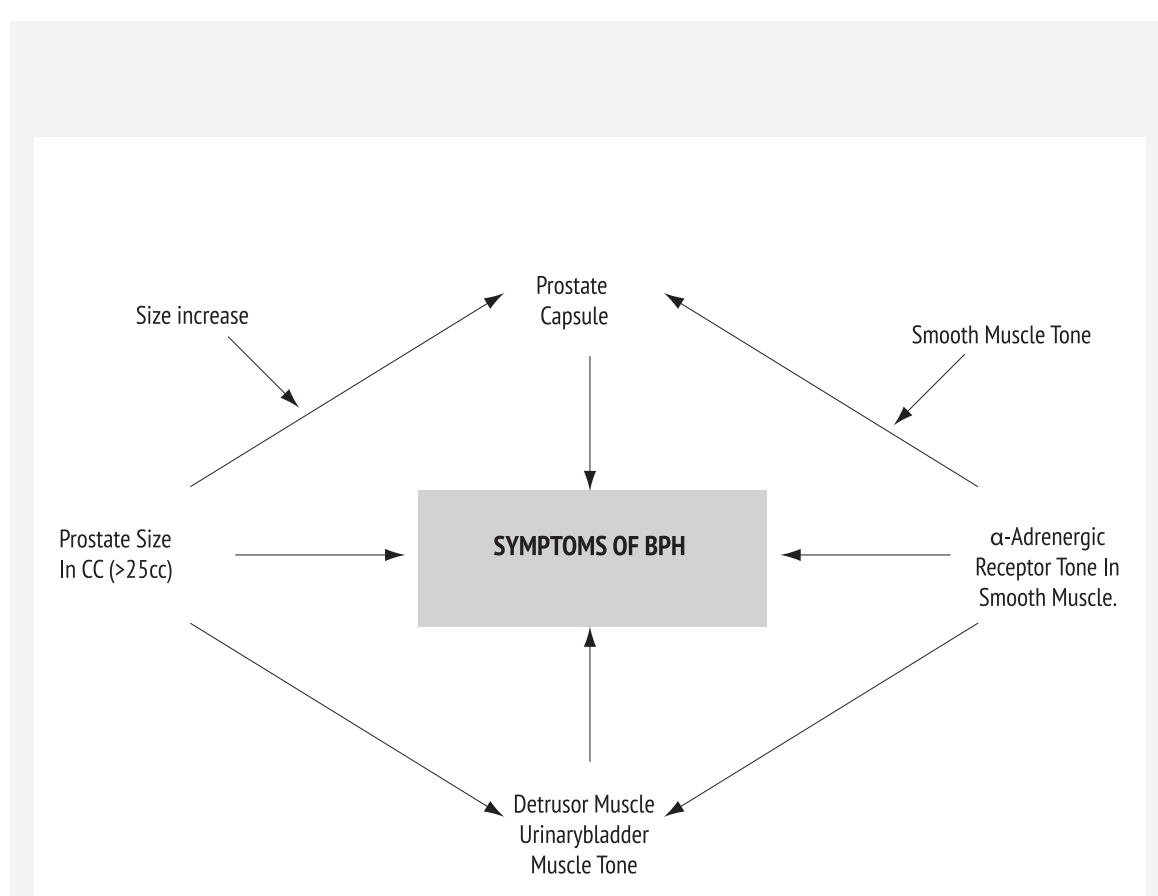

Fig. I: Factors That Lead To Symptoms Of Benign Prostate Hyperplasia( BPH)

\section{SELECTIVE BLOCKADE a1a and a1d RECEPTORS}

Reduced Detrusor muscle instability

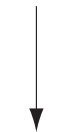

Decreases urgency, frequency incontinence

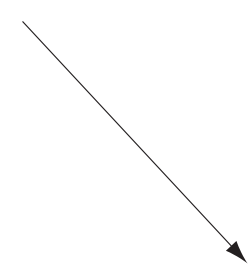

IMPROVED SYMPTOM OF BPH

Ref.Drugs 2002;62 (1):135-137

Fig. 2: Mechanism Of Action Of Uroselective $\alpha 1$-Blockers

factor in the development of symptoms of $\mathrm{BPH}$ (11) and the $\alpha$-adrenergic blockade is dependent on the percentage area density of the prostate smooth muscle (12). The number of these receptors increase with the prostatic size. Consequently, benign prostatic enlargement has more receptors compared to normal sized prostate $(13,14)$. The clinical application of this uroselectivity has resulted in the use of alfuzocin and tamsulocin in the initial treatment of BPH before progression to acute retention and need for surgery.

Both drugs are effective in treatment of symptoms (LUTS) and improving peak urinary flow rate ( $\mathrm{Q}$ max) by causing relaxation of the smooth muscles of the prostate gland and bladder neck (15). They do not however affect the prostatic volume (7).

Tamsulocin has a rapid onset of action of one week while alfuzocin works within 2-3 weeks. Alfuzosin additionally improves the sex drive in elderly men with erectile dysfunction (7)

The maximum improvement is felt at 6 months (24 weeks) but may continue to 18 months (64 weeks) (9). The effects of these $\alpha$-blockers are greater in younger patients $(<60 \mathrm{yrs})$ and smaller prostate volumes (<30cc). Treatment failure is associated with the baseline prostate volumes (larger than 40cc), prostate specific antigen (PSA) levels and drugs compliance (9).

Five $\alpha$-reductase inhibitors (ARIs) form the other group of drugs in the initial treatment of BPH. The two iso-enzymes, $5 \alpha$-reductase 1 and 2, convert testosterone to its active form, dihydrotestosterone (DHT).

DHT activates the androgen receptor complexes that lead to nuclear transcription factors that promote the proliferation and growth of the prostatic cells. $5 \alpha$-reductase iso-enzyme type 1 is less concentrated in the prostate cells but abundant in liver 
and skin while $5 \alpha$-reductase iso-enzyme type 2 is in abundance in the prostate and genital tissues (15).

Finasteride was the first $5 \alpha$-reductase (isoenzyme 2) inhibitor to be studied. Adverse effects associated with its use including reduction of libido and gynaecomastia encouraged the discovery of a second 5 ARI, dutasteride (14). The latter, launched in 2002, is a potent dual inhibitor and inhibits both the iso-enzyme type 1 and type 2 .

The suppression of DHT by dutasteride is 95\% within the first month of its use. This suppression is reversible when dutasteride is discontinued (16). The intra prostatic DHT suppression has been reported as $89.3 \%$ at 2 weeks, $92.4 \%$ at 4 weeks, and $98.9 \%$ at 4 months (17).

This reduction of DHT levels has a significant effect on prostatic volume (PV) and reduction reaches 95\% clearance in six (6) months in blood. The prostatic volume shrinkage is about $24-25 \%$ by the 2 nd year of continuous taking of the drug dutasteride (18). This leads to symptoms and flow rate improvement by over $65 \%$ with effect maintained over 4 years.

Once an enlarged prostate becomes symptomatic then $\mathrm{BPH}$ progression continues to worsen resulting in AUR and BPH related surgery.

The objectives of medical therapy therefore include (i) to treat early disease before symptoms progress to severe forms and avoid AUR/BPH related surgery (ii) to slow down the progression if any (iii) to avoid complications resulting from surgery for the $\mathrm{BPH}$ and (iv) to allow patients choices when they demand medical therapy. 5 ARIs fit these objectives.

They control both symptoms of BPH and disease progression to AUR and need for surgery. The men at risk of disease progression benefit the most such as those with prostatic volume $>30 \mathrm{cc}$, PSA of $>1.5 \mathrm{ng} / \mathrm{ml}$ and $<10.0$ $\mathrm{ng} / \mathrm{ml}$, Qmax of less than $12 \mathrm{mls} /$ second, ages over 60 years and symptoms severity greater than 7 (19). The low adverse effects (AE's) in long-term use of dutasteride as a dual inhibitor of $5 \alpha$ reductase iso enzymes $(1,2)$ makes it to be tolerable and acceptable to patients economically and with good compliance, it gives good quality of life according to international prostatic symptom score(IPSS), quality of life(QOL) (20) .

\section{Combination Treatment}

Combination drugs medical therapy combines the best of the 5 alpha reductase dual inhibition and alpha-adrenergic blockade for $\mathrm{BPH}$ treatment (15). At the onset of treatment, the 5-alpha reductase inhibitors and $\alpha$-blockers have a combined effect on the symptoms severity reduction, the peak flow rate (Qmax), prostate volume reduction (>30cc). Further benefits of long-term use are reduction of risk for $\mathrm{BPH}$ progression to AUR and BPH-related surgery.

The key factors in combination drug therapy are the duration of treatment, (over four years for dutasteride and 6 months for $\alpha$-blockers).

The maximal effect of alpha blockers is felt at six months. It is therefore possible to withdraw $\alpha$-blocker drugs after this period without affecting the progressive improvement of symptoms. This allows dutasteride to be continued for longer time(21).

The landmark medical therapy of prostatic symptoms (MTOPs) trial demonstrated over 4 years of the combination of type 2 specific 5 ARI, finasteride and the alpha blocker doxasin was more effective than either agent alone in reducing overall clinical progression (22).

The CombAT (Avodart plus Tamsulosin) trial, 4-year, global, multicenter, randomized, double blind, parallel group study enrolled 4328 men with moderate and severe symptoms of $\mathrm{BPH}$ and prostate enlargement: (patients were 50 years and older with prostate volume $30 \mathrm{~cm}+$ and PSA > $1.5 \mathrm{ng} / \mathrm{ml}$ ).

The two year analysis showed combination treatment was associated with greater symptoms reduction at 3 months versus dutasteride; greater at 9 months versus 
Combination Drug Therapy for Benign Prostatic Hyperplasia (BPH) Oliech J.S.

tamsulosin. The peak flow rate improved versus dutasteride and Tamsulosin from 6 months. The 4-year CombAT data supported the long term use in men with moderate to severe lower urinary tract symptoms due to $\mathrm{BPH}$ and prostate enlargement with greater symptom benefit than either monotherapy $(22,23)$. (Table 1$)$.

In conclusion, $\mathrm{BPH}$ is a universal problem in elderly men with significant impact in their quality of life.

Drug combination medical therapy for BPH is the best first line choice to improve symptoms and prevent progression. Surgery is left for those who do not show imrprovement or those with severe symptomatology.

\section{Acknowledgement}

To Susan Murima for typing the manuscript and Hada Achieng for the sketching of diagrams and figures.

\section{References}

1. Barry M J, Fowler F J, Jr O'Leary M P The American Urological Association symptom score index for BPH J Urol 1992; 148: 1549-57

2. Barry M J, Epidemiology and natural history of benign prostate hyperplasia. Urol Clin North Am. 1990; 17:495-506

3. Barry M J, Williford W O, Chang Y et al. Benign prostat- ic hyperplasia specific health status measures in clinical research; how much change in the American Urological Association symptom index the BPH impact index is perceptible to patients. J Urol 1995;154:1770-4

4. Berry S J, Coffey DS, Walsh PC, et al. The development of human benign prostatic hyperplasia with age. J Urol 1994; 132: 474-79

5. Caine $M$, Pfan A, Peddbers $S$. The use of alpha adrenergic blockers in benign prostatic obstruction. Br J Urol $1976 ; 48: 255-63$

6. Caine M, Pfan A, Peddbers S. Adrenergic and cholinergic receptors in human prostate, prostate capsule and bladder neck. Br J Urol 1975: 47:193

7. Jardin A, Besadonn H, Delauche-Cavalier MC, Attah P (BPH-ALF Group) Alfuzocin for treatment of benign prostatic hypertrophy. Lancet 1991; 337: 1457-61

8. Oliech J.S. Treatment of benign prostate hyperplasia at Mater Hospital Nairobi. East Afr Med J 2007; 84: 556560

9. Jardin A, Besadonn H, Delauche-Cavalier MC, Attali P, (BPH-ALF Group) Long term treatment of benign prostatic hypertrophy with alfuzocin: A 12-18 month assessment. Br J Urol 1993; 72: 615-20

10. Lukacs M, McCarthey C,Grange JC.Long term QOL in patients with BPH treated with alfuzocin. Eur Urol 
$1993 ; 24: 34$

11. Lepor H, Auerbach S, Puras-Bac Z A, et al. A randomized placebo controlled multicentre study of the efficacy and safety of terazocin in treatment of benign prostate hyperplasia. J Urol 1992; 148:1467-74

12. Lepor $\mathrm{H}$, and the Tamsulocin Investigator Group.Long term evaluation of tamsulonin in benign prostatic hyperplasia: Placebo controlled double blind extension of phase III trial. Tamsulocin investigor Group. Urology 1998; 51: 901-6

13. Lepor H, Transulocin Investigator Group: Clinical evaluation of tamsulocin; a prostate selective alpha antagonist. J Urol 1995; 153: 274(A)

14. Lepor $\mathrm{H}$, Williford $\mathrm{W} \mathrm{O}$, Barry M J et al (VACOUP). The impact of medical therapy on bothersome effects due to symptoms, quality of life and global outcome; and factors predicting response. J Urol 1998; 160:1358-67

15. Abrams P Benign prostatic hyperplasia poorly correlated with symptoms. Br Med J 1993; 307:201

16. Abrams P, Schulman C C, Vaage S (European Transulocin Study Group): Tamsulocin, a selective $\alpha$-adrenoreceptors antagonist: randomized controlled trial in patients with benign prostatic obstruction (symptomatic BPH). Br J Urol 1995; 76: 325-336

17. Andriole GL, Kirby R. Safety and tolerabilityof dual 5alpha-redutase inhibitor dutasteride in treatment of benign prostatic hyperplasia. Eur Urol 2003;44:82-8

18. Schulman C C. Long term aspect of medical treatment of benign prostatic hyperplasia. Eur Urol 2001;40(Suppl.6):7-12.

19. Kirby R S Clinical uroselectivity of Alfuzocin in the treatment of benign prostatic hyperplasia. Eur Urol 1998; 33: 19-27

20. Roehborn CG , Boyle P,Nickel JC et al. Efficacy and safety of dual inhibitor of 5 alpha-reductase type 1 and 2 (dutasteride) in men with benign prostatic hyperplasia.Urology 2002;60:434-41.

21. Barkin J,Guimarae M, Jacobi G et al. Alpha-blocker therapy can be withdrawn in the majority of men following inial combination therapy with the dual 5 alpha -reductase inhibitor dutastride. Eur Urol 2003;44:46261.

22. Roehborn CG, McNicholas T. European Urology Supp.2003;2:13-19

23. Roehborn CG, Boyle P, Berner D et al.Serum prostatic specific antigen and prostate volume predict the long term changes in symptoms and flow rates; results of 4 years randomized tial comparing 5alpha reductase finasteride and placebo ( PLESS study) Urology 2001;58:58-210. 